\title{
Preparation and Charactrisation of 1,3-dipolar cycloaddition of nitrones with 4-amino antipyrene
}

https://doi.org/10.32792/utq/utj/vol11/4/5

\section{Dakhil Z. Mutlaq and Raad J. Ali \\ Department of chemistry, College Of Education for pure \\ Sciences, University of Basrah}

\begin{abstract}
:
Some nitrones (1-5), derived from N-phenyl hydroxylamine with substituted benzaldehyde such as (3-chlorobenzaldehyde, 4-chlorobenzaldehyde, 3nitrobenzaldehyde, 4-florobenzaldehyde, 2-nitrobenzaldehyde). In subsequent 1,3-dipolar cycloaddition reactions of nitrones with 4-amino antipyrene give isoxazolidines (6-10), They have been identified by ${ }^{1} \mathrm{HNMR}$, ${ }^{13}$ CNMR, IR and Mass spectra
\end{abstract}

keyword: nitrones, isoxazolidines, 1,3-dipolar cycloaddition, dipolarophile , heterocycles, benzaldehyde, $\mathrm{N}$-phenyl hedroxyl amine, 4-amino antipyrene.

\section{Introduction:}

Nitrones were discovered by E.Becmann in the 1880s by $\mathrm{N}$-alkylation of oximes $^{1}$. In the 1960s the high-yielding synthesis of isoxazolidines were discovered by Cope, Lebel, Brown, Delpierre and Huisgen. The reactions of nitrones dipoles play an important role in the history of cycloaddition reaction. The 1,3-dipolar cycloaddition also known as the Huisgen cycloaddition $^{2}$ is a classic reaction in organic chemistry consisting of the reaction of dipolarophile with a 1,3-dipolar compound that allows the production of various five-membered heterocycles ${ }^{3}$. High specificity 


\section{University of Thi-Qar Journal Vol.11 No.4 DEC 2016}

Web Site: https://jutq.utq.edu.iq/index.php/main Email: journal@jutq.utq.edu.iq

stereoselectivity associated with these reactions make them synthetically important ${ }^{4-6}$. The 1,3-dipolar cycloaddition reaction was proposed to proceed through a concerted mechanism ${ }^{7}$. Figuer 1.

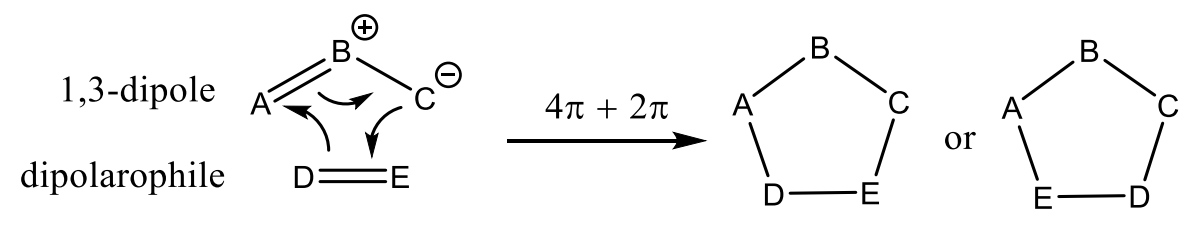

Fiuger 1: Generalized 1,3-dipolar cycloaddition reaction

For the concerted mechanism, the cycloaddition involves a reaction between a species containing four electrons in a three orbital array in the 1,3-dipole with the two electrons of the dipolarophile. The cycloaddition between these species is referred to as a $[4 \pi+2 \pi]$ cycloaddition. Most of dipolarophile are alkenes $^{8}$, alkynes ${ }^{9}$ and molecules possessing related hetero atom functional groups (such as carbonyls ${ }^{10,11}$ and nitriles ${ }^{12,13}$ ). Both inter and intra molecular nitrone and alkene cycloaddition reaction have received attention of heterocycles of biological interest ${ }^{14-16}$.

The arguments for the alternative are based on Sustmann's molecular orbital perturbation theory ${ }^{17}$ which can be simply illustrated by using the frontier $\pi$ MO's of the 1,3-dipole, the transition state of the cycloaddition, and the dipolarophile (Figure 2).

1,3-Dipole

T.S.

Dipolarophile
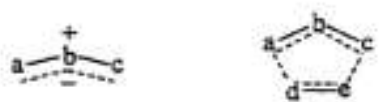

$\mathrm{d}=$

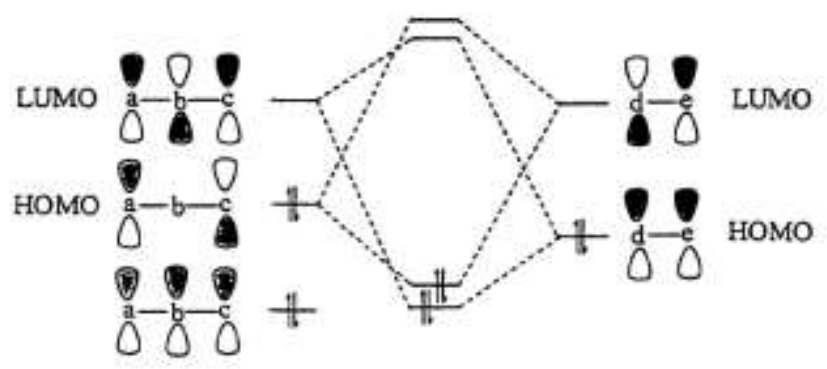




\section{University of Thi-Qar Journal Vol.11 No.4 DEC 2016}

Web Site: https://jutq.utq.edu.iq/index.php/main Email: journal@jutq.utq.edu.iq

Figure 2: The frontier $\pi$-MO's 1,3-dipolar cycloaddition

There are two sets of HOMO-LUMO pairs. When the energy gap between one pair of the HOMO-LUMO is much smaller than the other pair, the electron flow would be unidirectional, in a concerted process. Otherwise the electron flow would be two directional: from HOMO (1,3-dipole) to LUMO (dipolarophile) and back from HOMO (dipolarophile) to LUMO (1,3-dipole) to from the two new $\sigma$-bonds. The two step cycloaddition process will take place only under one of the following conditions: either the atomic coefficient at one end of the 1,3-dipole is much smaller than at the other or a strong steric hindrance exists at one end of the 1,3-dipole. ${ }^{18}$ The reactivity and regioselectivity in 1,3-dipolar cycloaddition reactions can also be approached by using Figure 2 .

\section{Results and Discussion}

The nitrones (1-5) used in this study were easily prepared from the corresponding aldehyde with $\mathrm{N}$-phenyl hedroxyl amine. ${ }^{19,20}$ Scheme 1.

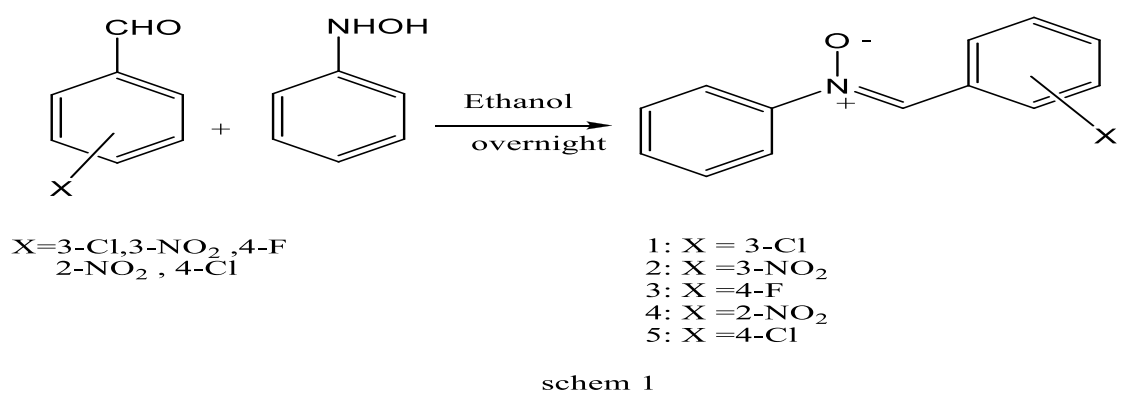

The cycloaddition of nitrones (1-5) with 4-amino antipyrene were carried out by refluxing (48-72 h) in dry toluene at $110{ }^{\circ} \mathrm{C}$ to give isoxazolidines (6-10). In all cases, the compounds were purified by column chromatography ${ }^{21}$ allowed the isolation of pure compounds. Scheme 2 . 
Web Site: https://jutq.utq.edu.iq/index.php/main Email: journal@jutq.utq.edu.iq

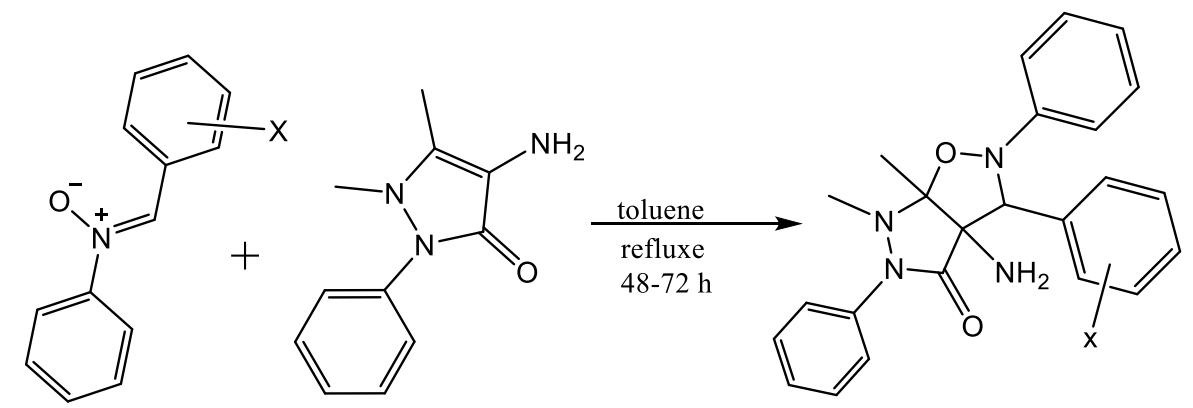

schem 2

$$
\begin{aligned}
& \text { 6: } \mathrm{X}=3-\mathrm{Cl} \\
& \text { 7:X }=3-\mathrm{NO}_{2} \\
& \text { 8: } \mathrm{X}=4-\mathrm{F} \\
& \text { 9:X }=2-\mathrm{NO}_{2} \\
& \text { 10: } \mathrm{X}=4-\mathrm{Cl}
\end{aligned}
$$

The obtained isoxazolidines were characterized spectroscopically. The formation of the cycloadducts was established by the ${ }^{1} \mathrm{H} N M R,{ }^{13} \mathrm{C} N M R$, and IR spectroscopy. The ${ }^{1} \mathrm{H}$ NMR spectrum of isoxazolidines (6-10) showed a singlet at $\delta 9.49-10.02$ ppm for $\left(\mathrm{NH}_{2}\right)$, a multiplet at $\delta$ 7.09-7.79 ppm for the aromatic protons, including a singlet at $\delta 7.36-7.39 \mathrm{ppm}$ for ( ${ }^{\mathrm{N}-\mathrm{C}}$-ph $)$, singlet at 2.99-3.19 ppm for $\left(\mathrm{N}-\mathrm{CH}_{3}\right)$ and a singlet at $\delta$ 2.3-2.47 ppm for $\left(\mathrm{C}-\mathrm{CH}_{3}\right)$. See figures (3-7), More detail of the ${ }^{1} \mathrm{H}$ NMR data analysis for compounds (6-10) can be seen in Table (1).

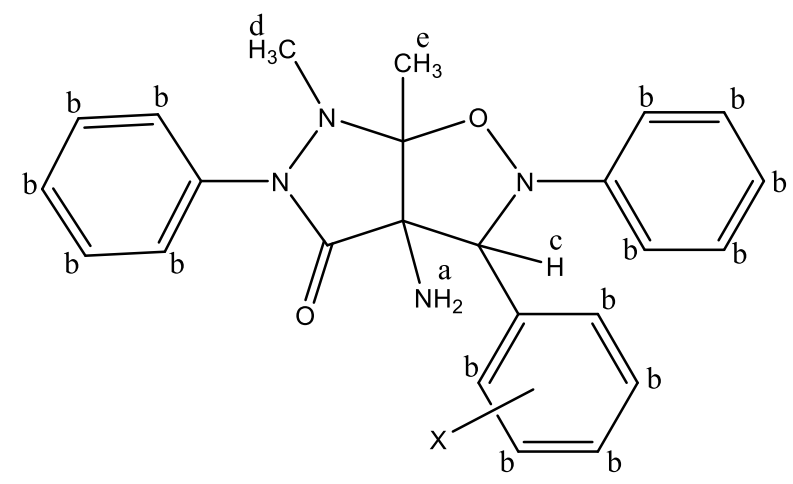


University of Thi-Qar Journal Vol.11 No.4 DEC 2016

Web Site: https://jutq.utq.edu.iq/index.php/main Email: journal@jutq.utq.edu.iq

Table (1): ${ }^{1} \mathrm{H}$ NMR data analysis of isoxazolidines (6-10)

\begin{tabular}{|c|c|c|r|c|c|c|}
\hline Comp. & $\mathrm{X}$ & $\mathrm{H}_{\mathrm{a}}$ & \multicolumn{1}{c|}{$\mathrm{H}_{\mathrm{b}}$} & $\mathrm{H}_{\mathrm{c}}$ & $\mathrm{H}_{\mathrm{d}}$ & $\mathrm{H}_{\mathrm{e}}$ \\
\hline 6 & $3-\mathrm{Cl}$ & $9.72(\mathrm{~s}$, & $7.37-7.79(\mathrm{~m}$, & $7.36(\mathrm{~s}$, & $3.16(\mathrm{~s}$, & $2.4(\mathrm{~s}$, \\
& & $2 \mathrm{H})$ & $14 \mathrm{H})$ & $1 \mathrm{H})$ & $3 \mathrm{H})$ & $3 \mathrm{H})$ \\
\hline 7 & $3-\mathrm{NO}_{2}$ & $9.7(\mathrm{~s}, 2 \mathrm{H})$ & $7.32-7.91(\mathrm{~m}$, & $7.38(\mathrm{~s}$, & $3.17(\mathrm{~s}$, & $2.4(\mathrm{~s}$, \\
& & & $14 \mathrm{H})$ & $1 \mathrm{H})$ & $3 \mathrm{H})$ & $3 \mathrm{H})$ \\
\hline 8 & $4-\mathrm{F}$ & $9.57(\mathrm{~s}$, & $7.16-8.48(\mathrm{~m}$, & $7.37(\mathrm{~s}$, & $3.02(\mathrm{~s}$, & $2.3(\mathrm{~s}$, \\
& & $2 \mathrm{H})$ & $14 \mathrm{H})$ & $1 \mathrm{H})$ & $3 \mathrm{H})$ & $3 \mathrm{H})$ \\
\hline 9 & $2-\mathrm{NO}_{2}$ & $9.73(\mathrm{~s}$, & $7.099-$ & $2.47(\mathrm{~s}$, & $3.14(\mathrm{~s}$, & $7.39(\mathrm{~s}$, \\
& & $2 \mathrm{H})$ & $7.85(\mathrm{~m}$, & $3 \mathrm{H})$ & $3 \mathrm{H})$ & $1 \mathrm{H})$ \\
& & & $14 \mathrm{H})$ & & & \\
\hline 10 & $4-\mathrm{Cl}$ & $10.02(\mathrm{~s}$, & $7.32-8.14(\mathrm{~m}$, & $2.46(\mathrm{~s}$, & $3.19(\mathrm{~s}$, & $7.39(\mathrm{~s}$, \\
& & $2 \mathrm{H})$ & $14 \mathrm{H})$ & $3 \mathrm{H})$ & $3 \mathrm{H})$ & $1 \mathrm{H})$ \\
\hline
\end{tabular}

The ${ }^{13} \mathrm{C}$ NMR spectrum of isoxazolidines (6-10) included singlet at $\delta 160$ $180 \mathrm{ppm}$ for the $(\mathrm{C}=\mathrm{O})$, at $\delta 125-160 \mathrm{ppm}$ for aromatic carbons. See figures (8-11). More detail of the ${ }^{13} \mathrm{C}$ NMR data analysis for compounds (6-10) can be seen in Table (2).

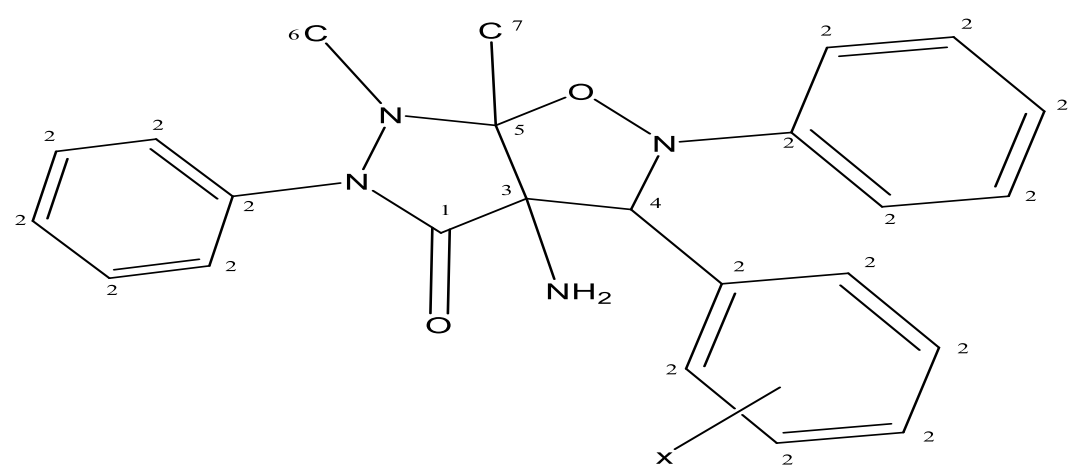


University of Thi-Qar Journal Vol.11 No.4 DEC 2016

Web Site: https://jutq.utq.edu.iq/index.php/main Email: journal@jutq.utq.edu.iq

Table (2): ${ }^{13} \mathrm{C}$ NMR data analysis of isoxazolidines (6-10)

\begin{tabular}{|c|c|c|c|c|c|c|c|c|}
\hline Comp. & $\mathrm{X}$ & $\mathrm{C} 1$ & $\mathrm{C} 2$ & $\mathrm{C} 3$ & $\mathrm{C} 4$ & $\mathrm{C} 5$ & $\mathrm{C} 6$ & $\mathrm{C} 7$ \\
\hline 6 & $3-\mathrm{Cl}$ & $160 \delta$ & $130-$ & 118.29 & 124.75 & $134 \delta$ & 35.98 & 10.35 \\
& & & $155 \delta$ & $\delta$ & $\delta$ & & $\delta$ & $\delta$ \\
\hline 7 & $3-\mathrm{NO}_{2}$ & $160 \delta$ & $124-$ & 118.2 & 124.77 & 134.9 & $35.9 \delta$ & $10.4 \delta$ \\
& & & $157 \delta$ & $\delta$ & $\delta$ & $\delta$ & & \\
\hline 8 & $4-\mathrm{F}$ & $160 \delta$ & $124-$ & 117.65 & 121.7 & 134.68 & 35.74 & 10.37 \\
& & & $160 \delta$ & $\delta$ & $\delta$ & $\delta$ & $\delta$ & $\delta$ \\
\hline 9 & $2-\mathrm{NO}_{2}$ & $160 \delta$ & $124.3-$ & 117.62 & 12169 & 134.68 & 35.73 & 10.35 \\
& & & $153.2 \delta$ & $\delta$ & $\delta$ & $\delta$ & $\delta$ & $\delta$ \\
\hline
\end{tabular}

The IR spectrum included a peak at $3059-3150 \mathrm{~cm}^{-1}$ for the $\mathrm{NH}_{2}$ stretch and the sharp peak at $1647-1651 \mathrm{~cm}^{-1}$ for $\mathrm{C}=\mathrm{O}$ stretch. See figures (16-20). The mass spectrum of compounds (6-10) gave the correct molecular ion. See figures (12-15).

The synthesis of isoxazolidines (6-10) were confirmed using ${ }^{1} \mathrm{H} \mathrm{NMR},{ }^{13} \mathrm{C}$ NMR, Mass spectrometry and IR.

The results described above are in general agreement with the frontier orbital treatment of 1,3-dipolar cycloadditions. ${ }^{22}$ This view suggests the formation of the carbon substituted isoxazolidines regioselectivity. As the ionization potential and the electron affinity of the alkene increase (i.e. as the HOMOLUMO level decrease in energy) there is an increasing tendency towards the formation of a regioisomeric mixture of adducts. In this cycloaddition reaction, the carbon-carbon and carbon-oxygen bond formation in the transition state may not happen in a synchronous manner. 


\section{University of Thi-Qar Journal Vol.11 No.4 DEC 2016}

Web Site: https://jutq.utq.edu.iq/index.php/main Email: journal@jutq.utq.edu.iq

\section{Conclusion:}

In conclusion, 1,3-dipolar cycloaddition reaction of some nitrones (1-5) to 4amino antipyrene to give new isoxazolidines (6-10).

\section{4- Experimental}

4.1 General methods:

Melting points were determined using a Gallenkamp melting point apparatus. Proton and carbon NMR spectra were recorded on a Bruker DRX 500 Advance spectrometer at $500 \mathrm{MHz}$ and $125 \mathrm{MHz}$, respectively, using deuterated solvents and TMS as an internal standard

Chemical shifts are reported as $\delta$ values in ppm. Infrared spectra were obtained an FT-IR-1600 Perkin-Elmer spectrophotometer thin layer chromatography (TLC) was performed on aluminum sheets silica gel from merk. Column chromatography was carried out using Merck silica gel (230400 mesh). The TLC spots were visualized in UV and $\mathrm{I}_{2}$. Mass spectra recorded on High-resolution mass spectra were recorded on an ESI-TOF Mariner Spectrometer (Perspective Biosystem)

\subsection{Preparation Methods}

\subsubsection{Preparation of the nitrones (1-5)}

The $\mathrm{N}$-phenyl hedroxyl amine was prepared from nitrobenzene according to ref ${ }^{26}$ and $\alpha$-aryl-N-phenyl nitrones (1-5) from the respective substituted benzaldehyde and $\mathrm{N}$-phenyl hedroxyl amine according to ref ${ }^{23-27}$.

4.2.2 Preparation of the isoxazolidines (6-10)

General procedure for cycloaddition of nitrones (1-5) to 4-amino antipyrene. To a stirred solution of the nitrones (1-5) $(5 \mathrm{mmole})$ in dry toluene $(50 \mathrm{ml})$ was added to 4 -amino antipyrene ( 5 mmole) and the solution was heated at reflux $(48-72 \mathrm{~h})$. The resulting mixture was evaporated under reduced 


\section{University of Thi-Qar Journal Vol.11 No.4 DEC 2016}

Web Site: https://jutq.utq.edu.iq/index.php/main Email: journal@jutq.utq.edu.iq

pressure. The crude product was purified by column chromatography on silica gel eluting to give pure isoxazolidines (6-10).

4.2.2.1 3 $\alpha$-amino-3-(4-chlorophenyl)-6,6a-dimethyl-2,5- diphenyltetrahydro$2 \mathrm{H}$-pyrazolo[4,3-d]isoxazol-4(5H)-one (6): the product (6) was isolated by column chromatography on silica gel eluting with benzene $\backslash$ methanol(8:2) as a brown solid product in $60 \%$ yield, m.p $=254-255 \mathrm{C}^{\circ}$.

IR: $1651 \mathrm{~cm}^{-1}(\mathrm{C}=\mathrm{O}), 3100 \mathrm{~cm}^{-1}\left(\mathrm{NH}_{2}\right), 1455 \mathrm{~cm}^{-1}(\mathrm{C}=\mathrm{C}), 1300 \mathrm{~cm}^{-1}(\mathrm{C}-\mathrm{N})$; ${ }^{1} \mathrm{H}$ NMR : $\delta 9.75 \mathrm{ppm}(\mathrm{s}, 2 \mathrm{H}), 7.32-7.79 \mathrm{ppm}(\mathrm{m}, 15 \mathrm{H}$ aromatic) including singlet at $7.36 \mathrm{ppm}$ for (ph-CH), $3.16 \mathrm{ppm}(\mathrm{s}, 3 \mathrm{H}), 2.4 \mathrm{ppm}(\mathrm{s}, 3 \mathrm{H})$; ${ }^{13} \mathrm{C}$ NMR: $\delta 160 \mathrm{ppm}(\mathrm{C}=\mathrm{O}), 155-130 \mathrm{ppm}$ ( C aromatic), 134 ppm (C-O), 124.75 ppm (C-ph) , 35.98 ppm $\left(\mathrm{N}-\mathrm{CH}_{3}\right), 10.35$ ppm $\left(\mathrm{C}-\mathrm{CH}_{3}\right)$; Mass: $\mathrm{m} \backslash \mathrm{z}=$ $434[\mathrm{M}]^{+}$.

4.2.2.2 3 $\alpha$-amino-3-(3-chlorophenyl)-6,6a-dimethyl-2,5-diphenyltetrahydro2H-pyrazolo[4,3-d]isoxazol-4(5H)-one(7): the product (7) was isolated by column chromatography on silica gel eluting with benzene $\backslash$ methanol(8:2) as a brown solid product in $51 \%$ yield, m.p $=186 \mathrm{C}^{\circ}$.

IR: $1647 \mathrm{~cm}^{-1}(\mathrm{C}=\mathrm{O}), 3150 \mathrm{~cm}^{-1}\left(\mathrm{NH}_{2}\right), 1423 \mathrm{~cm}^{-1}(\mathrm{C}=\mathrm{C}), 1307 \mathrm{~cm}^{-1}(\mathrm{C}-\mathrm{N})$;

${ }^{1} \mathrm{H}$ NMR: $\delta 9.7 \mathrm{ppm} \quad(\mathrm{s}, 2 \mathrm{H}), 7.32-7.91 \mathrm{ppm}(\mathrm{m}, 15 \mathrm{H}$ aromatic) including singlet at $7.3 \mathrm{ppm}$ for ph-CH, $3.17 \mathrm{ppm}(\mathrm{s}, 3 \mathrm{H}), 2.4 \mathrm{ppm}(\mathrm{s}, 3 \mathrm{H}) ;{ }^{13} \mathrm{C}$ NMR: $\delta$ 160 ppm (C=O), 157-124 ppm (C aromatic), 118.2 ppm $\left(\mathrm{C}-\mathrm{NH}_{2}\right), 134.9$ ppm $\left(\mathrm{C}-\mathrm{CH}_{3}\right), 124.77$ ppm (C-ph), 35.9 ppm (N-CH$), 10.4 \mathrm{ppm}(\mathrm{C}-\mathrm{CH} 3)$; Mass: $\mathrm{m} \backslash \mathrm{z}=434.3[\mathrm{M}]^{+}$.

4.2.2.3 3 $\alpha$-amino-6,6a-dimethyl-3-(3-nitrophenyl)2,5diphenyltetrahydro2H-pyrazolo[4,3-d]isoxazol-4(5H)-one(8): the product (8) was isolated by column chromatography on silica gel eluting with benzene $\backslash$ methanol(8:2) as a yellow solid product in $55 \%$ yield, m.p $=210-212 \mathrm{C}^{\circ}$. 


\section{University of Thi-Qar Journal Vol.11 No.4 DEC 2016}

Web Site: https://jutq.utq.edu.iq/index.php/main Email: journal@jutq.utq.edu.iq

IR: $1647 \mathrm{~cm}^{-1}(\mathrm{C}=\mathrm{O}), 3100 \mathrm{~cm}^{-1}\left(\mathrm{NH}_{2}\right), 1492 \mathrm{~cm}^{-1}(\mathrm{C}=\mathrm{C}), 1311 \mathrm{~cm}^{-1}(\mathrm{C}-\mathrm{N})$, $1023 \mathrm{~cm}^{-1}$ ( $\mathrm{NO}_{2}$ Asym), $1340 \mathrm{~cm}^{-1}\left(\mathrm{NO}_{2}, \mathrm{sym}\right)$;

${ }^{1} \mathrm{H}$ NMR : $\delta 9.57 \mathrm{ppm}(\mathrm{s}, 2 \mathrm{H}), 7.16-8.48 \mathrm{ppm}$ (m,15 H aromatic) including singlet at $7.37 \mathrm{ppm}$ for ph-CH, $3.02 \mathrm{ppm}(\mathrm{s}, 3 \mathrm{H}), 2.3 \mathrm{ppm}(\mathrm{s}, 3 \mathrm{H}) ;{ }^{13} \mathrm{C}$ NMR: $\delta 180 \mathrm{ppm}(\mathrm{C}=\mathrm{O}), 160-124.3 \mathrm{ppm}$ ( $\mathrm{C}$ aromatic),117.65 ppm $\left(\mathrm{C}-\mathrm{NH}_{2}\right)$, $134.68\left(\mathrm{C}-\mathrm{CH}_{3}\right), 121.7 \mathrm{ppm}(\mathrm{C}-\mathrm{ph}), 35.7 \mathrm{ppm}\left(\mathrm{N}-\mathrm{CH}_{3}\right), 10.37$ ppm (C$\mathrm{CH}_{3}$ ); Mass: $\mathrm{mlz}=[\mathrm{M}]^{+}$.

4.2.2.4 3 3 -amino-3-(4-fluorophenyl)-6,6a-dimethyl-2,5-diphenyltetrahydro2H-pyrazolo[4,3-d]isoxazol-4(5H)-one (9) yield 52\%, m.p=230- $231 \mathrm{C}^{\circ}$. IR: $1651 \mathrm{~cm}^{-1}(\mathrm{C}=\mathrm{O}), 3100 \mathrm{~cm}^{-1}\left(\mathrm{NH}_{2}\right), 1492 \mathrm{~cm}^{-1}(\mathrm{C}=\mathrm{C}), 1303 \mathrm{~cm}^{-1}$ ${ }^{1} \mathrm{H}$ NMR : $\delta 9.73 \mathrm{ppm}(\mathrm{s}, 2 \mathrm{H}), 7.9-7.85 \mathrm{ppm}$ (m,15H aromatic) including singlet at $7.39 \mathrm{ppm}$ for (ph-CH), $3.14 \mathrm{ppm}(\mathrm{s}, 3 \mathrm{H}), 2.47 \mathrm{ppm}(\mathrm{s}, 3 \mathrm{H})$; ${ }^{13} \mathrm{C}$ NMR: $\delta 160 \mathrm{ppm}(\mathrm{C}=\mathrm{O}), 153.2-124.3 \mathrm{ppm}$ ( $\mathrm{C}$ aromatic),117.62 ppm $\left(\mathrm{C}-\mathrm{NH}_{2}\right), 134.68 \mathrm{ppm}\left(\mathrm{C}-\mathrm{CH}_{3}\right), 121.69 \mathrm{ppm}(\mathrm{C}-\mathrm{ph}), 35.73 \mathrm{ppm}\left(\mathrm{N}-\mathrm{CH}_{3}\right)$, 10.37 ppm $\left(\mathrm{C}-\mathrm{CH}_{3}\right)$; Mass: $\mathrm{m} \backslash \mathrm{z}=418[\mathrm{M}]^{+}$.

4.2.2.5 $3 \alpha$-amino-6,6a-dimethyl-3-(2-nitrophenyl)-2,5-diphenyltetrahydro2H-pyrazolo[4,3-d]isoxazol-4(5H)-one(10): yield $60 \%, \mathrm{~m} . \mathrm{p}=209-210 \mathrm{C}^{\circ}$. IR: $1651 \mathrm{~cm} \mathrm{-1}(\mathrm{C}=\mathrm{O}), 3120 \mathrm{~cm}^{-1}\left(\mathrm{NH}_{2}\right), 1485 \mathrm{~cm}^{-1}(\mathrm{C}=\mathrm{C}), 1303 \mathrm{~cm}^{-1}(\mathrm{C}-\mathrm{N})$, $1519 \mathrm{~cm}^{-1}$ ( $\mathrm{NO}_{2}$ Asym.), $1357 \mathrm{~cm}^{-1}\left(\mathrm{NO}_{2}\right.$, sym.);

${ }^{1} \mathrm{H}$ NMR : $\delta 10.02 \mathrm{ppm}(\mathrm{s}, 2 \mathrm{H}), 7.32-8.14 \mathrm{ppm}(\mathrm{m}, 15 \mathrm{H}$ aromatic) including singlet at $7.39 \mathrm{ppm}$ for (ph-CH), $3.19 \mathrm{ppm}(\mathrm{s}, 3 \mathrm{H}), 2.46 \mathrm{ppm}(\mathrm{s}, 3 \mathrm{H})$; Mass: $\mathrm{m} \backslash \mathrm{z}=459[\mathrm{M}]^{+}$. 


\section{University of Thi-Qar Journal Vol.11 No.4 DEC 2016}

Web Site: https://jutq.utq.edu.iq/index.php/main Email: journal@jutq.utq.edu.iq

\section{References}

1- E.Beckmann, Ber.Dtsch. Chem. Ges.; (1890), 23, 3331.

2- R. Huisgen, Angew. Chem., Int. Ed. Engl.; (1963),10, pp 565-598

3- A. Padwa . 1,3-Dipolar Cycloaddition Chemistry Vols 1-2. Wiley Interscience, New York (1984).

4- M.K. Werner, M.J. de los Santos, and M. Steven; J.org.chem (1999), 64, pp 4865-4873

5- B.B. Barry , H. Lin; Am.chem.soc (1999), 121, pp 7778-7786.

6- R. Huisgen, In 1,3-Dipolar Cycloaddition Chemistry; A. Padwa, , Ed.; Wiley: New York, (1984); Vol. 1, pp 1-176

7- R.S. Menon, V. Nair , Molecular Sciences and Chemical Engineering, from Comprehensive Organic Synthesis II (2 ed), (2014), 4, pp 1281-1341

8- J.Malinina, T. Q. Tran, A. V. Stepakov, V. V. Gurzhiy, G. L. Starova,R. R. Kostikov, A. P. Molchanov; Tetrahedron Letters, (2014), 55 ,pp 36633666

9- M. Arnó, R.J. Zaragozá, L.R. Domingo; Tetrahedron Asymmetry, (2004), 15, pp1541-1549

10- W.Wang, R. T. Cassell, K.S. Rein; Tetrahedron Asymmetry, (2013), 24, pp1541-1549

11- M. M. Heravi, V. Zadsirjan; Tetrahedron: Asymmetry (2014), 25, pp1061-1090

12- D. Carmona , M. P. Lamata, F. Viguri, R. Rodríguez, F. J. Lahoz;

Tetrahedron: Asymmetry 20 (2009), 20, pp1197-1205

13- K.Grela, L.Konopski ;Tetrahedron, (2010), 66, pp3614-3622

14- K.V. Gothelf and K.A. Jorgensen; Chem.Rev., (1998), 98, pp 863-910

15-G. Broggini,G. Zecchi; Synthesis (1999), 17, 905

16-J.Mulzer,organic synthesis Highlights, Verlag Chemic,Weinheim. (1991) p77 


\section{University of Thi-Qar Journal Vol.11 No.4 DEC 2016}

Web Site: https://jutq.utq.edu.iq/index.php/main Email: journal@jutq.utq.edu.iq

17- R. Sustmann; Tetrahedron Let., (1971), 2717

18- R. Huisgen; Lectures in Heterocyclic Chem., (1987), 9, s-1.

19- K. Tadano, K. Hakuba, H. Kimura, Seiichiro Ogawa; J.org.chem. (1989), 54, pp 276-279

20- A. Dondoni, F.S.Merchan; synth.commun, (1994), 22, 2200

21- C. L. Varela, C. Amaral, E. T. Silva; European Journal of Medicinal Chemistry, (2014), 87, pp 336-345

22-SK. A. Ali, J.H. Khan and M.I.M. Wazeer. Tetrahedron, (1988), 44, 5911-5920.

23-H.H.Salman and N.N.Majeed.; J.Basrah Researches sciences (2013), 39, 99-111

24- J. J. Tufariello, In 1,3-Dipolar Cycloaddition Chemistry; A.Padwa, ,Ed.; J.Wiley \&S. Sons: New York, ( 1984); Vol. 2, Chapter 9.

25- K. B. Torssell, G. In Nitrile Oxides, Nitrones and Nitronates in Organic Synthesis; Feuer, H., Ed.;VCH: Weinheim, Germany, (1988).

26- R. C. F.Jones; J. N. Martin, In Synthetic Applications of 1,3-Dipolar Cycloadditions. Chemistry Toward Heterocycles and Natural Products; Padwa, A., Pearson, W. H., Eds; John Wiley \& Sons:Hoboken, NJ, (2003); Chapter 1.

27- P. N. Confalone, E. M. Huie; Org. React. (1988), 36,1. 


\section{University of Thi-Qar Journal Vol.11 No.4 DEC 2016}

Web Site: https://jutq.utq.edu.iq/index.php/main Email: journal@jutq.utq.edu.iq

$$
\begin{aligned}
& \text { تحضير ونتخيص ودر اسة الاضافة } \\
& \text { الحلقية 3,1- ثنائية القطب للنايترونات مع } \\
& \text { 4-أمينو انتي بايرين } \\
& \text { داخل زغير مطلق و رائد جميل علي } \\
& \text { قسم الكيمياء كلية التربية للعلوم الصرفة جامعة البصرة }
\end{aligned}
$$

حضرت بعض مركبات النايترونات (1-5) مشنقة من تفاعل ن- فنيل هيدروكسيل امين مع معوضـات البنز الدهايد ـ ضمن تفاعلات الاضافة الحلقية 3,1- ثنائية القطب للنايترونات مع 4-أمينو انتي بايرين ليعطي مركبات الازوكسازولدينات (6-10) وتم تعبينها باستخدام طيف بروتون وكاربون-13 للرنين النووي المغناطيسي وطيف الاشعة تحت الحمر اء وكذلك طيف الكتلة.

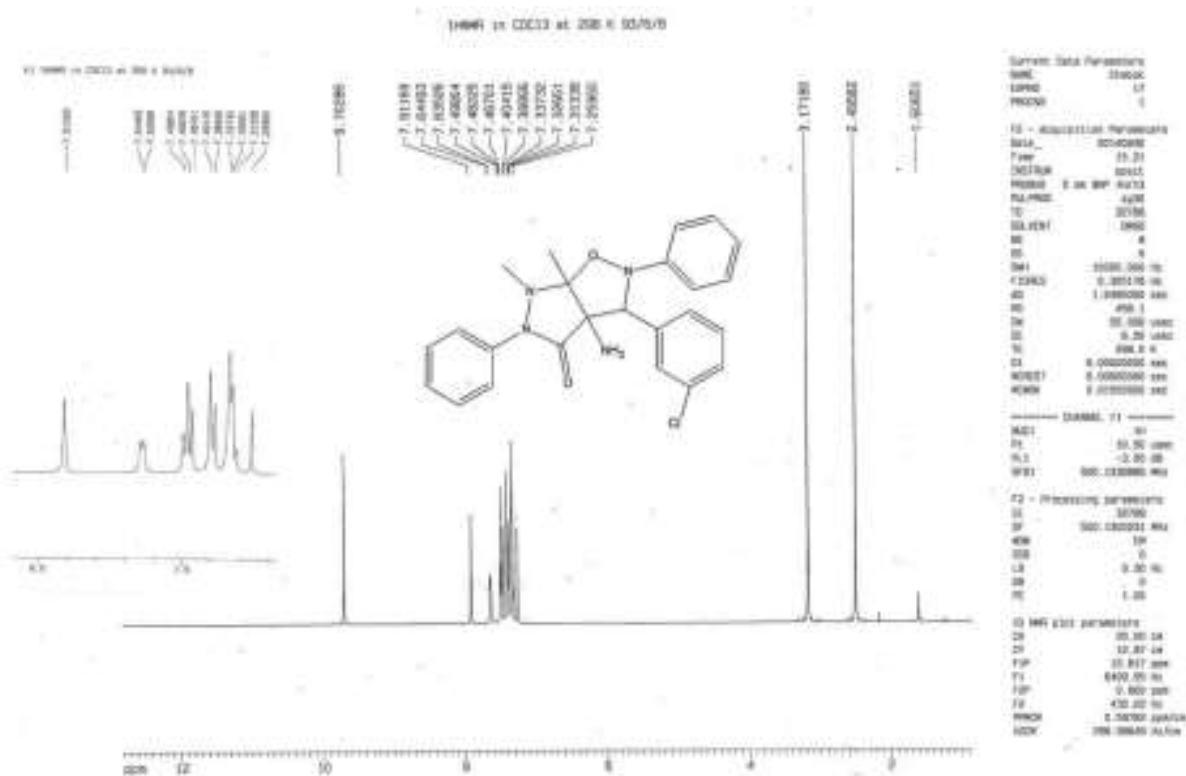

Fig. (3) ${ }^{1}$ HNMR for compound (6) 
Web Site: https://jutq.utq.edu.iq/index.php/main Email: journal@jutq.utq.edu.iq

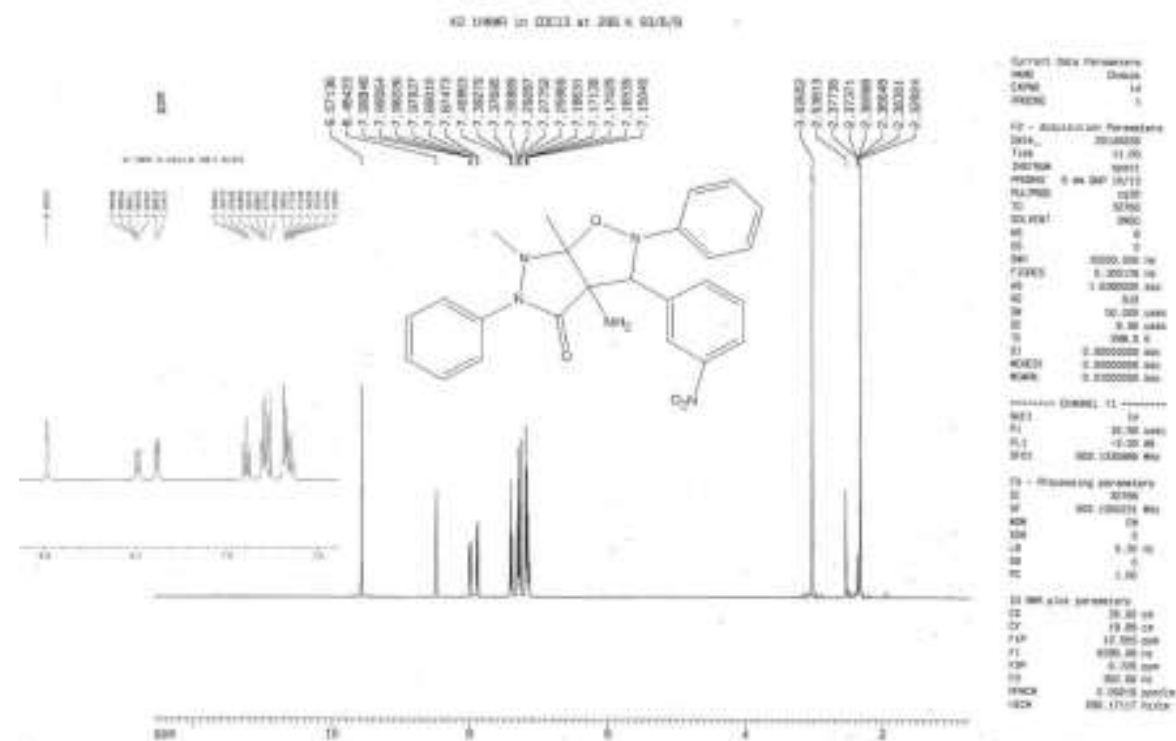

Fig. (4) ${ }^{1}$ HNMR for compound (7)

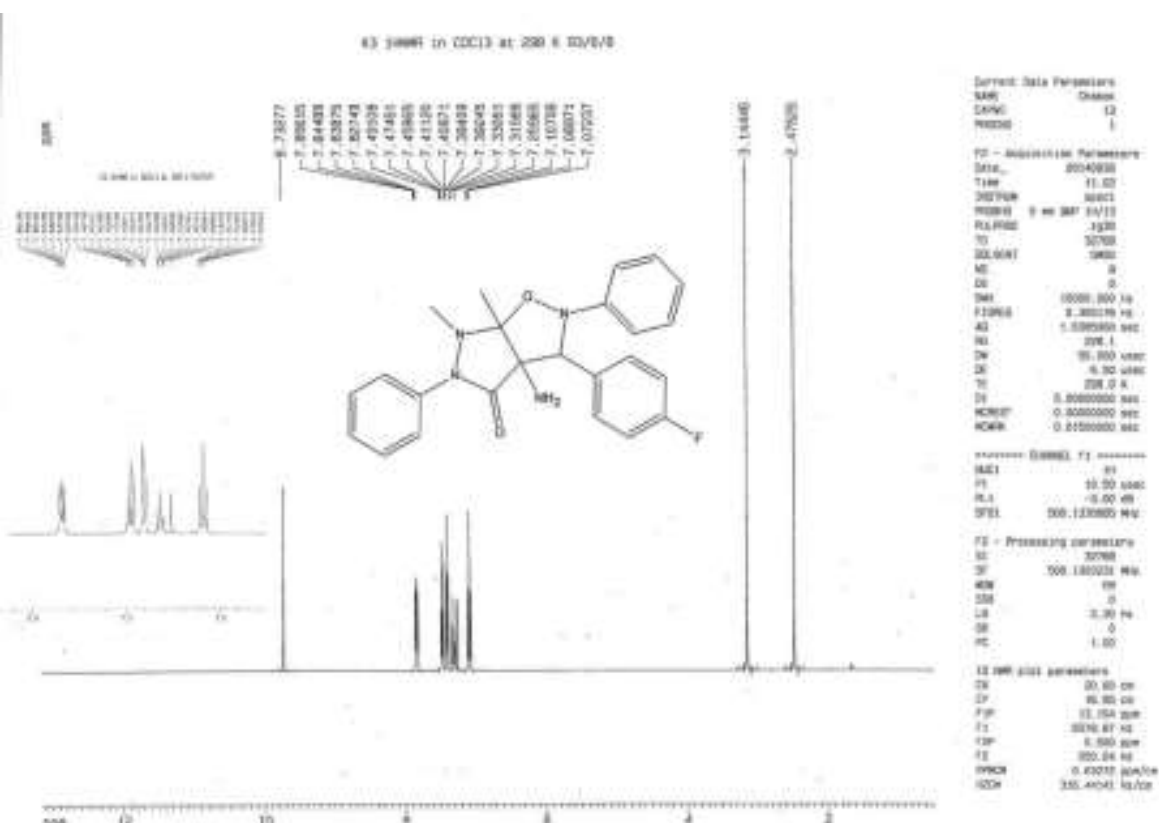

Fig. (5) ${ }^{1} \mathrm{HNMR}$ for compound (8) 
Web Site: https://jutq.utq.edu.iq/index.php/main Email: journal@jutq.utq.edu.iq
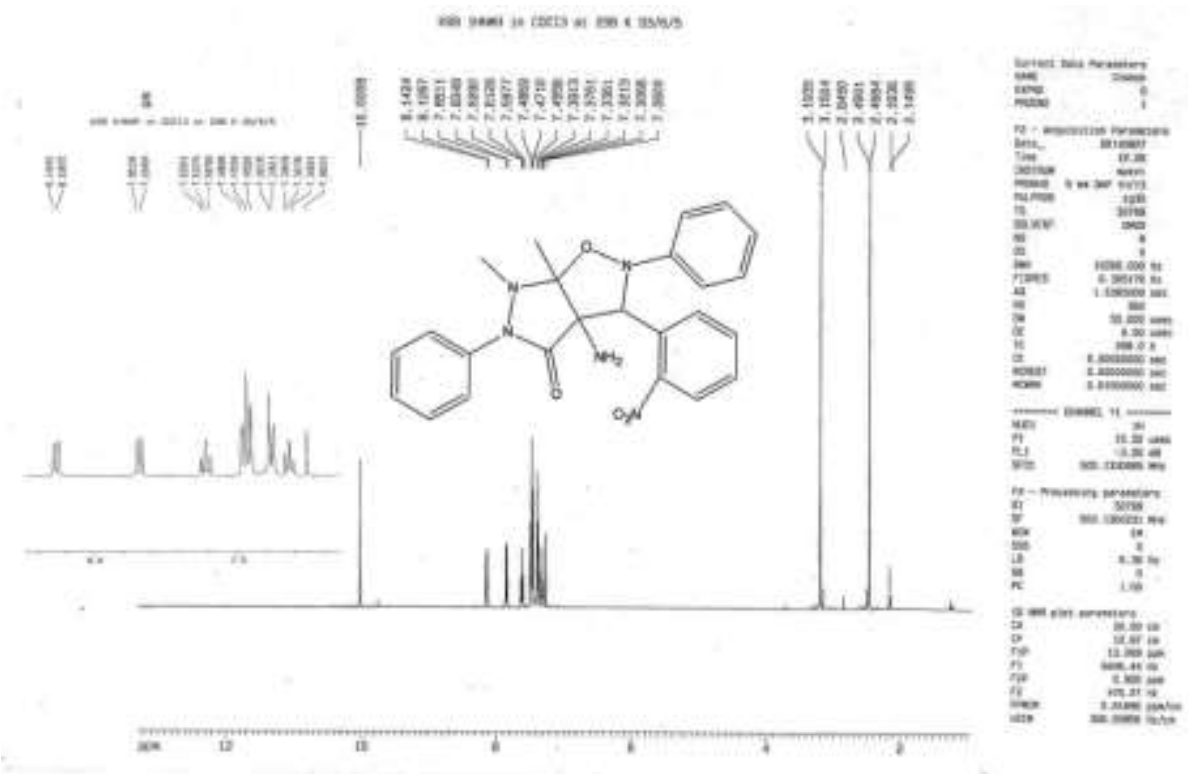

Fig. (6) ${ }^{1}$ HNMR for compound (9)

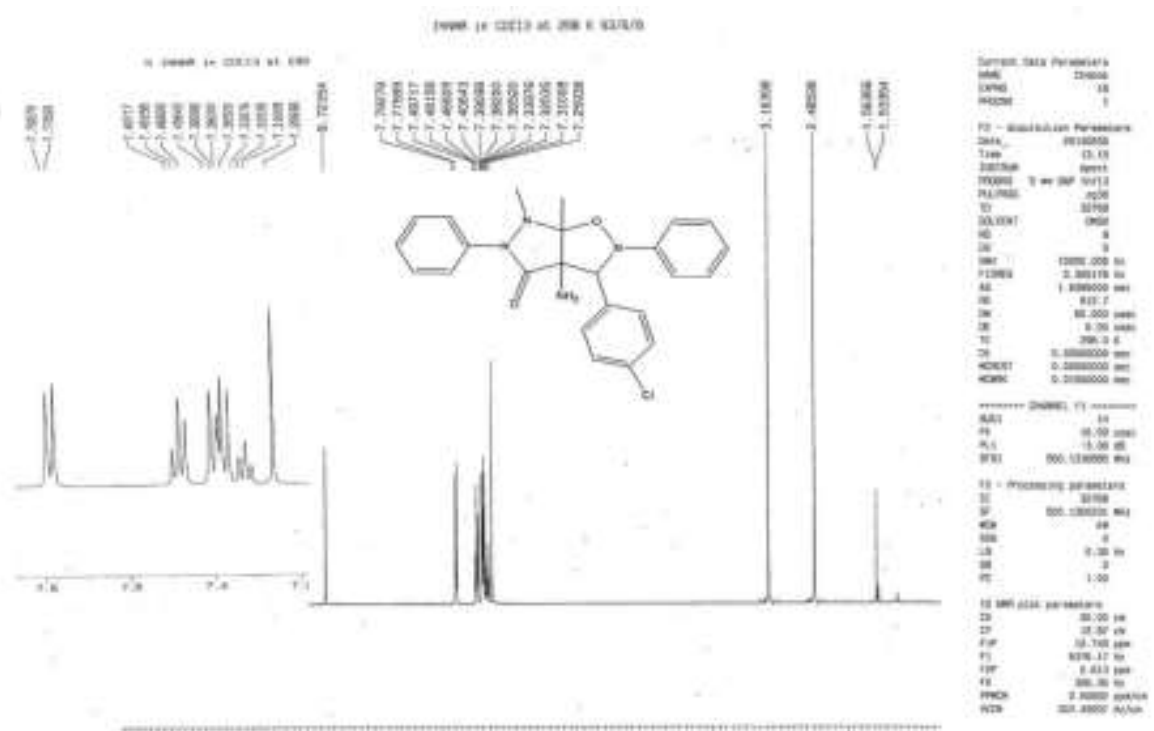

Fig. (7) ${ }^{1}$ HNMR for compound (10) 
University of Thi-Qar Journal Vol.11 No.4 DEC 2016

Web Site: https://jutq.utq.edu.iq/index.php/main Email: journal@jutq.utq.edu.iq

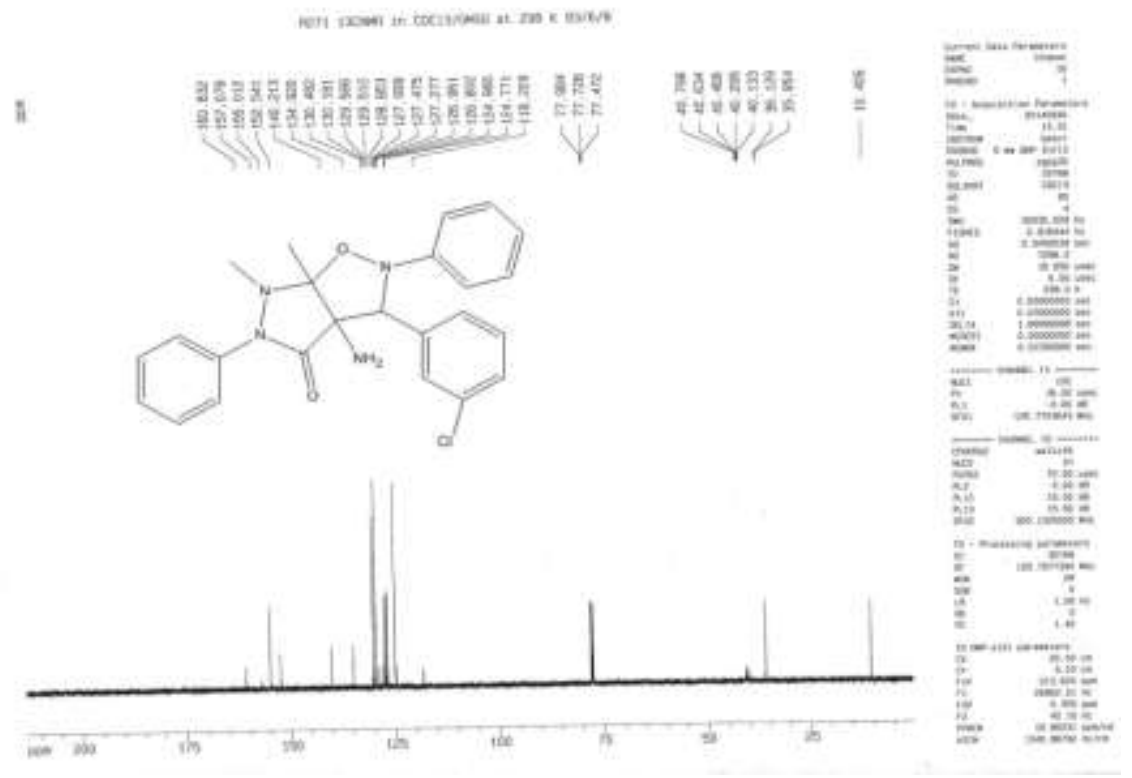

Fig. (8) ${ }^{13}$ CNMR spectrum for compound (6)

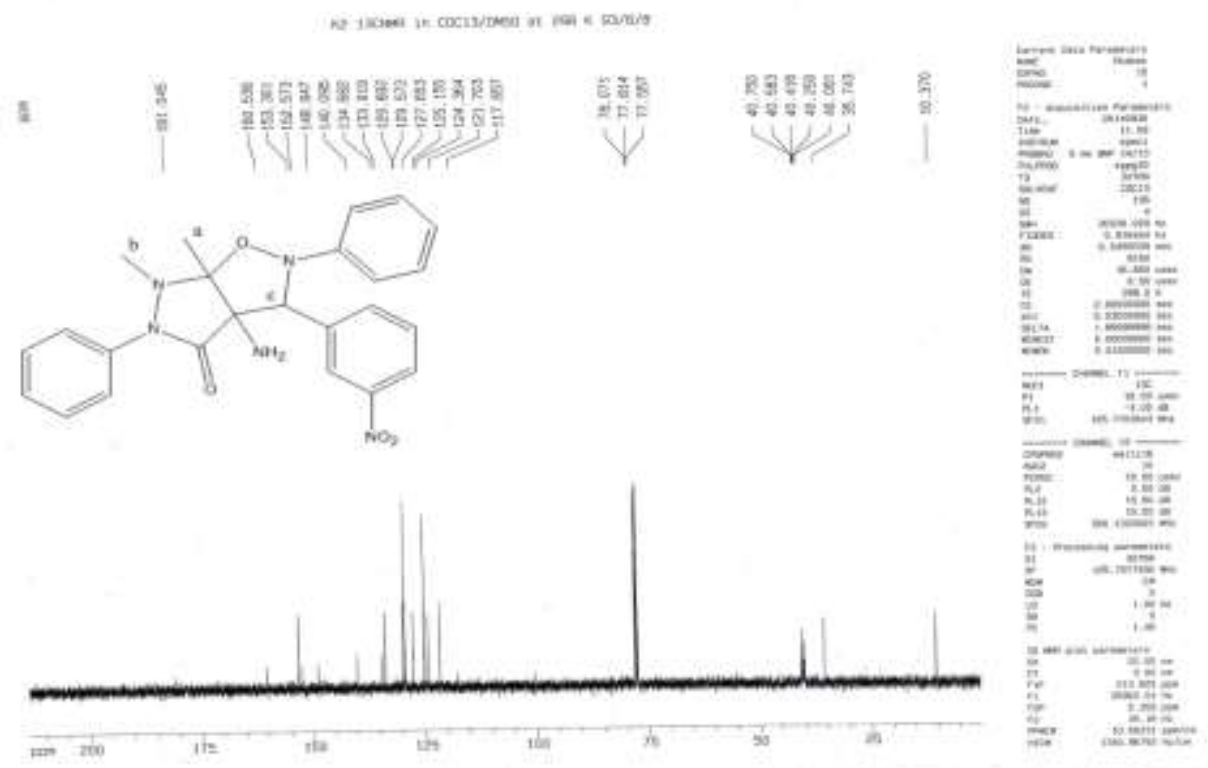

Fig.(9) ${ }^{13}$ CNMR spectrum for compound (7) 
University of Thi-Qar Journal Vol.11 No.4 DEC 2016

Web Site: https://jutq.utq.edu.iq/index.php/main Email: journal@jutq.utq.edu.iq

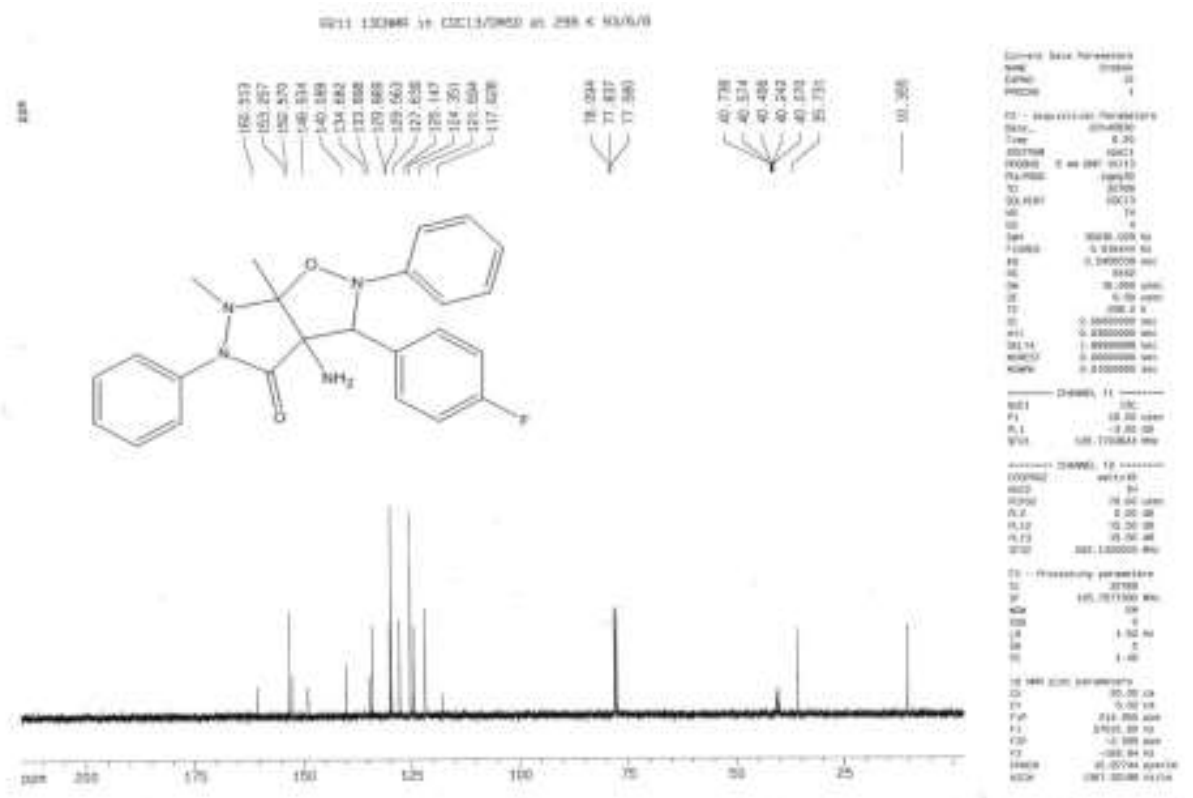

Fig. (10) ${ }^{13}$ CNMR spectrum for compound (8)

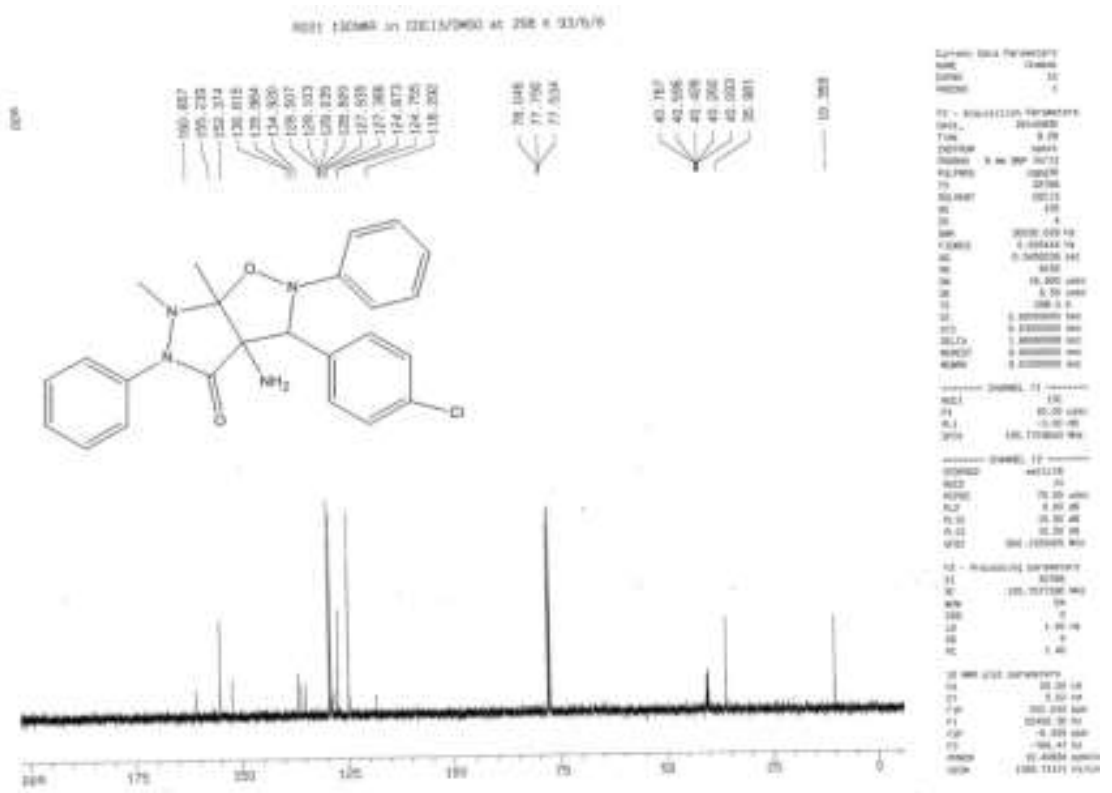

Fig. (11) ${ }^{13}$ CNMR spectrum for compound (10) 
University of Thi-Qar Journal Vol.11 No.4 DEC 2016

Web Site: https://jutq.utq.edu.iq/index.php/main Email: journal@jutq.utq.edu.iq

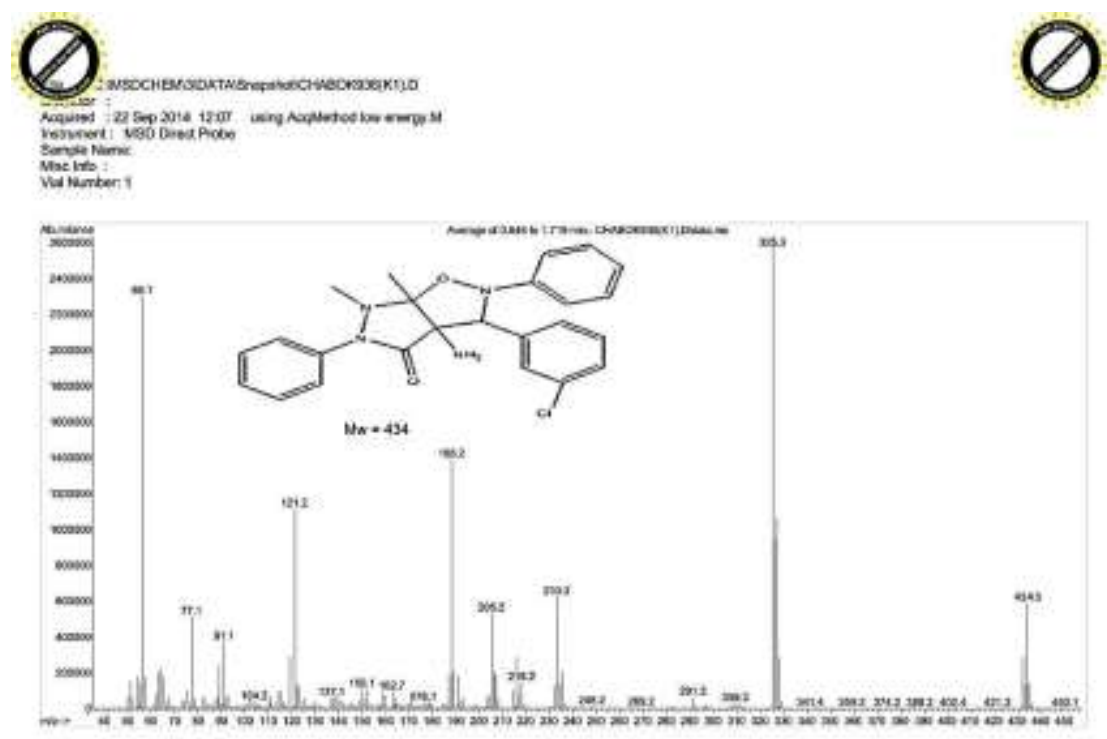

Fig. (12) Mass spectrum for compound (6)

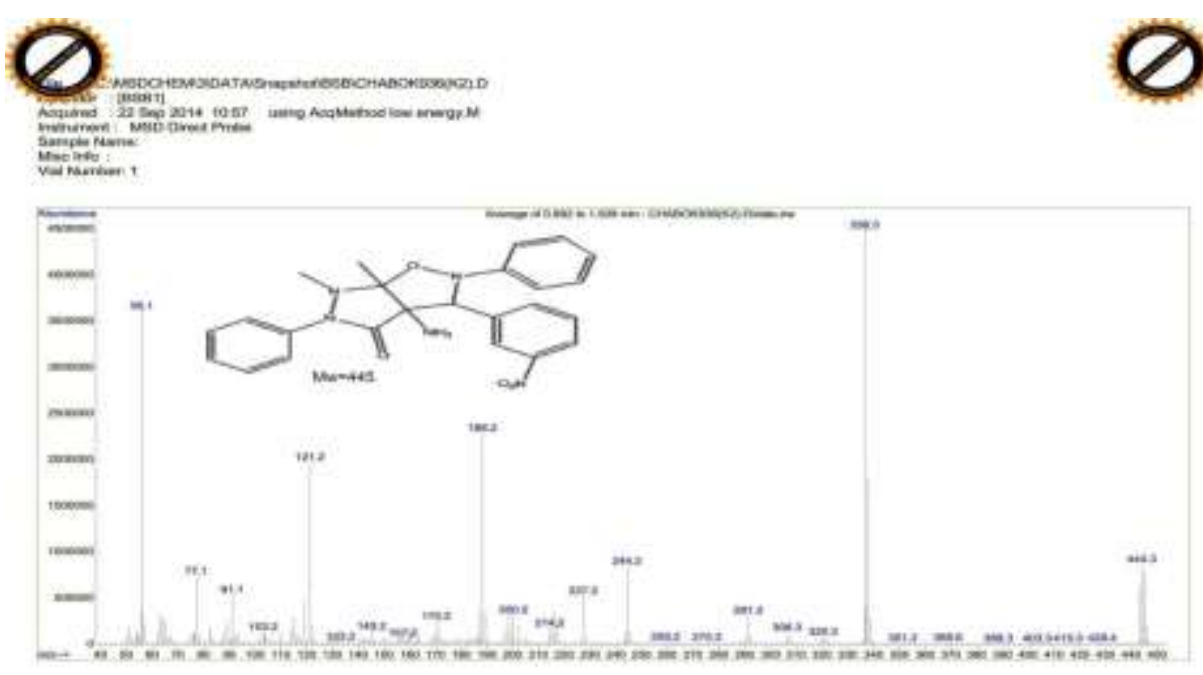

Fig. (13) Mass spectrum for compound (7) 
University of Thi-Qar Journal Vol.11 No.4 DEC 2016

Web Site: https://jutq.utq.edu.iq/index.php/main Email: journal@jutq.utq.edu.iq

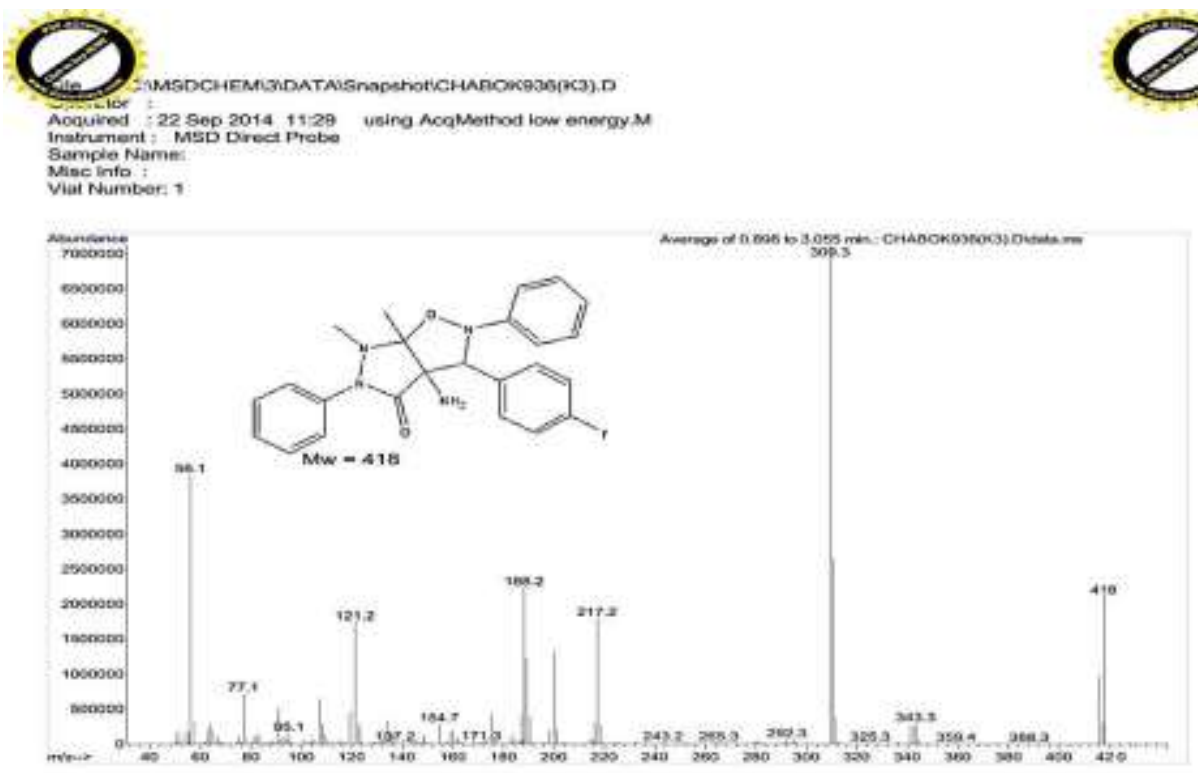

Fig. (14) Mass spectrum for compound (8)

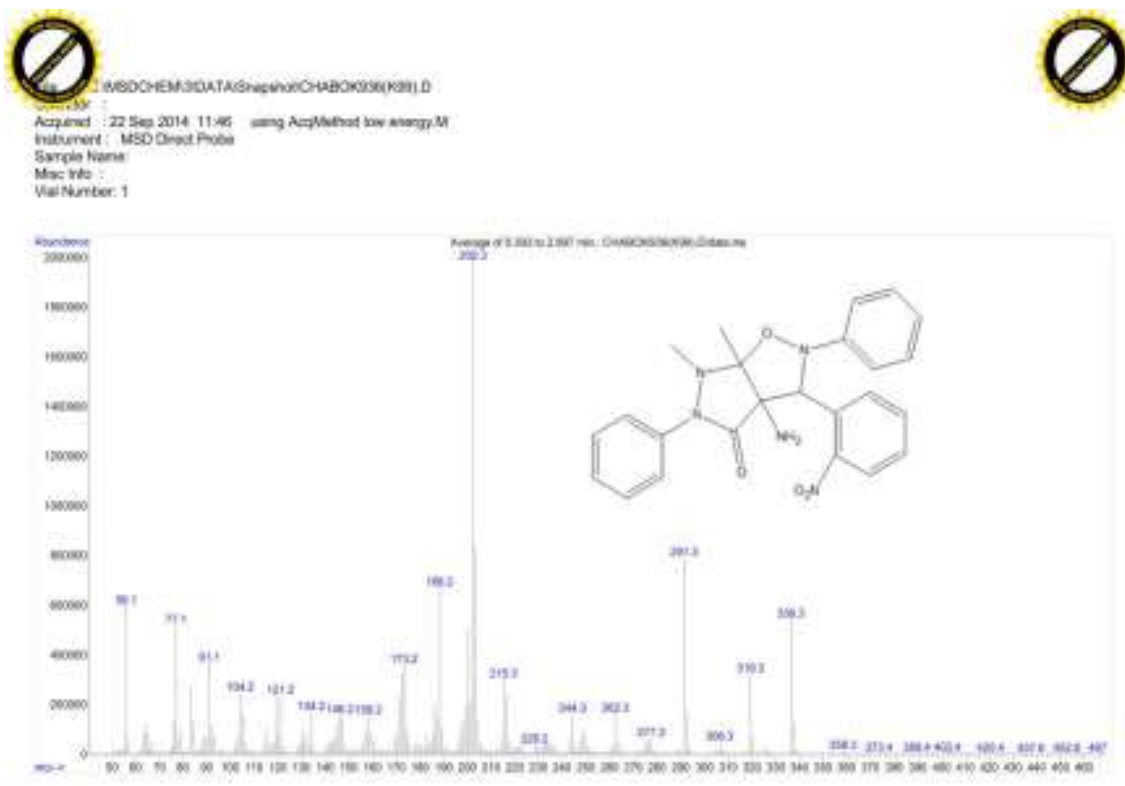

Fig. (15) Mass spectrum for compound (9) 
University of Thi-Qar Journal Vol.11 No.4 DEC 2016

Web Site: https://jutq.utq.edu.iq/index.php/main Email: journal@jutq.utq.edu.iq

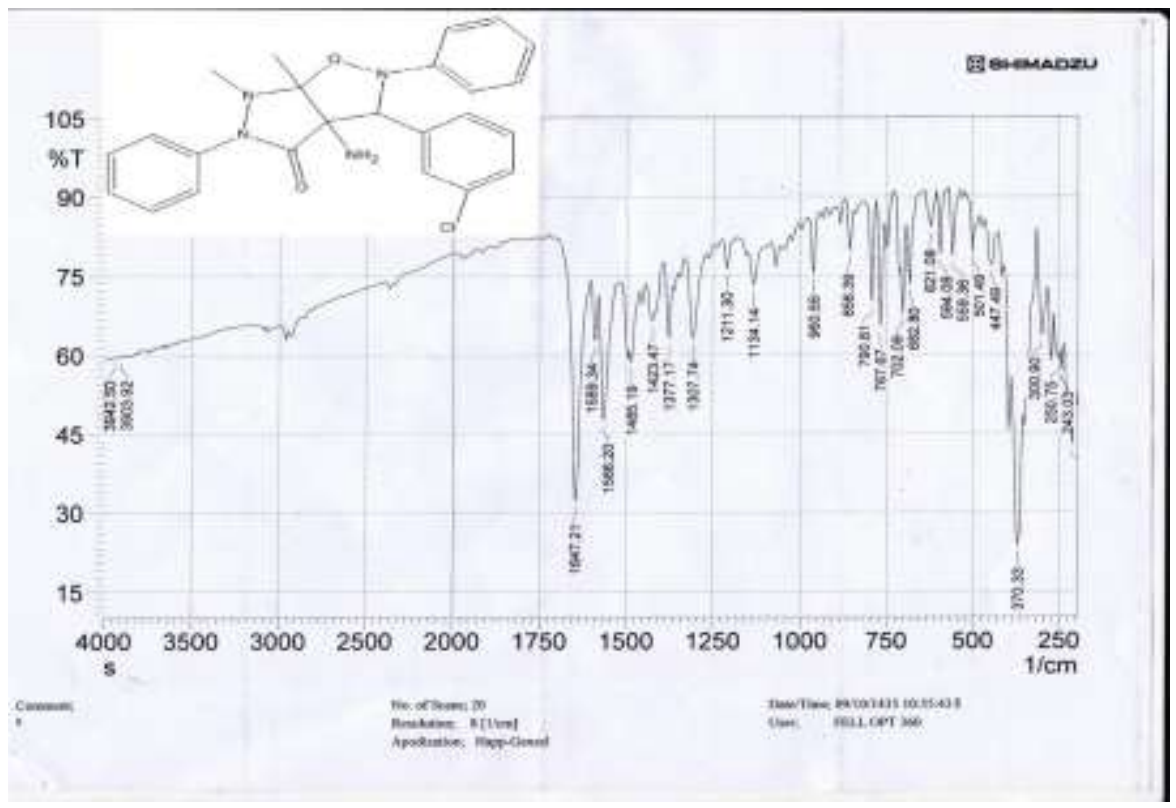

Fig. (16) IR spectrum for compound (6)

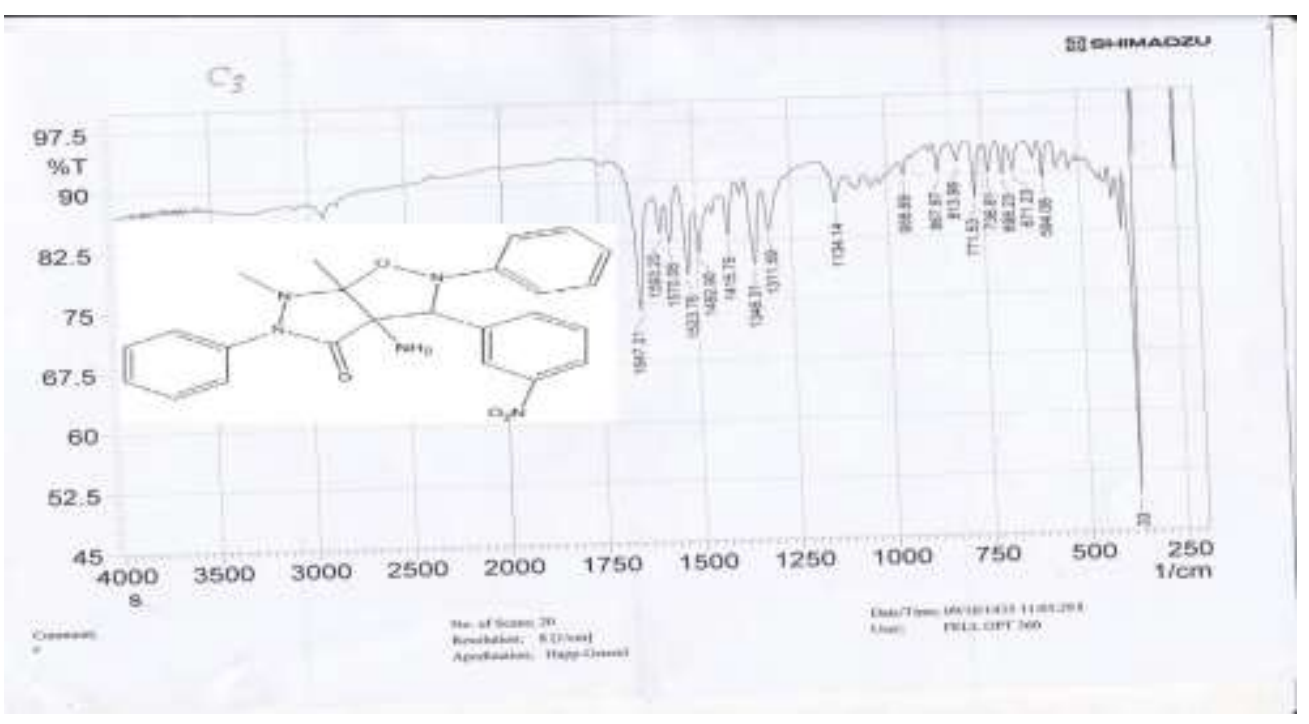

Fig. (17) IR spectrum for compound (7) 
University of Thi-Qar Journal Vol.11 No.4 DEC 2016

Web Site: https://jutq.utq.edu.iq/index.php/main Email: journal@jutq.utq.edu.iq

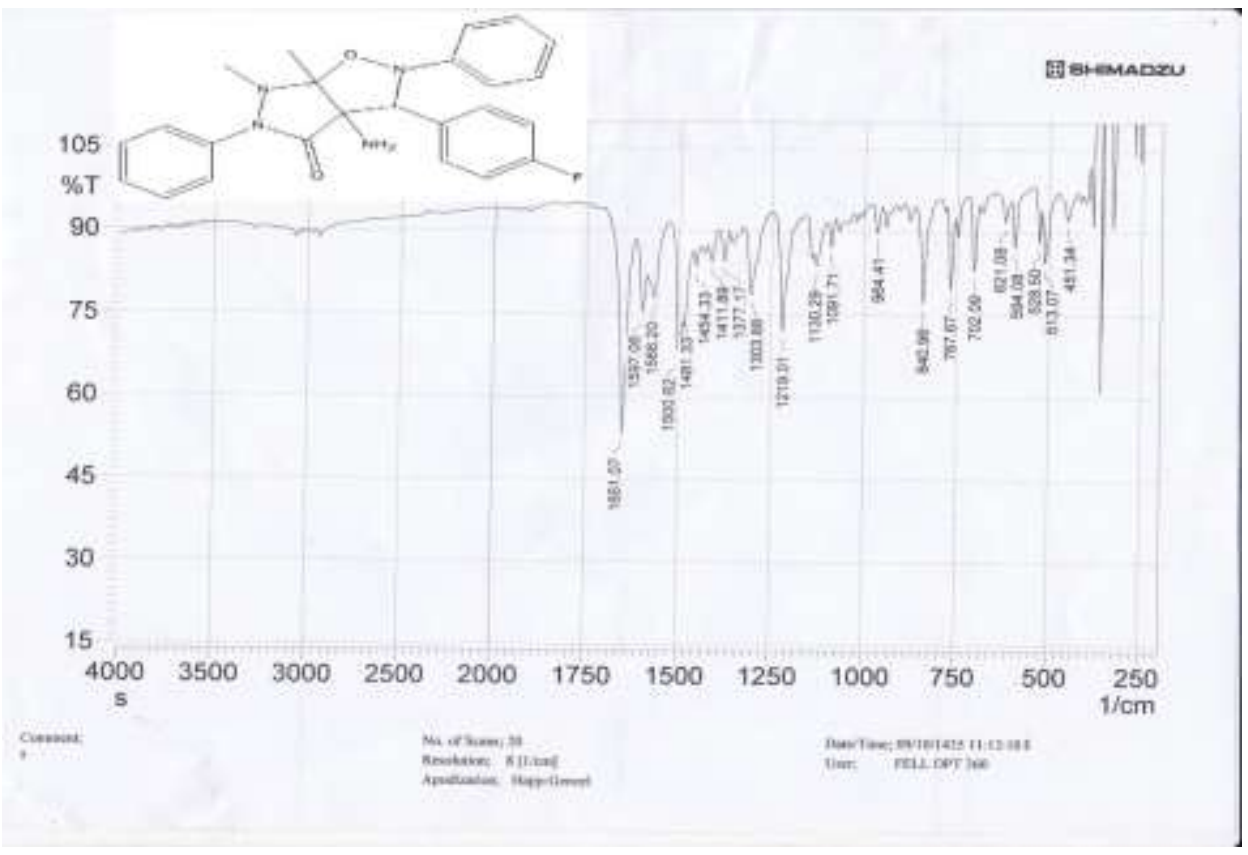

Fig. (18) IR spectrum for compound (8)

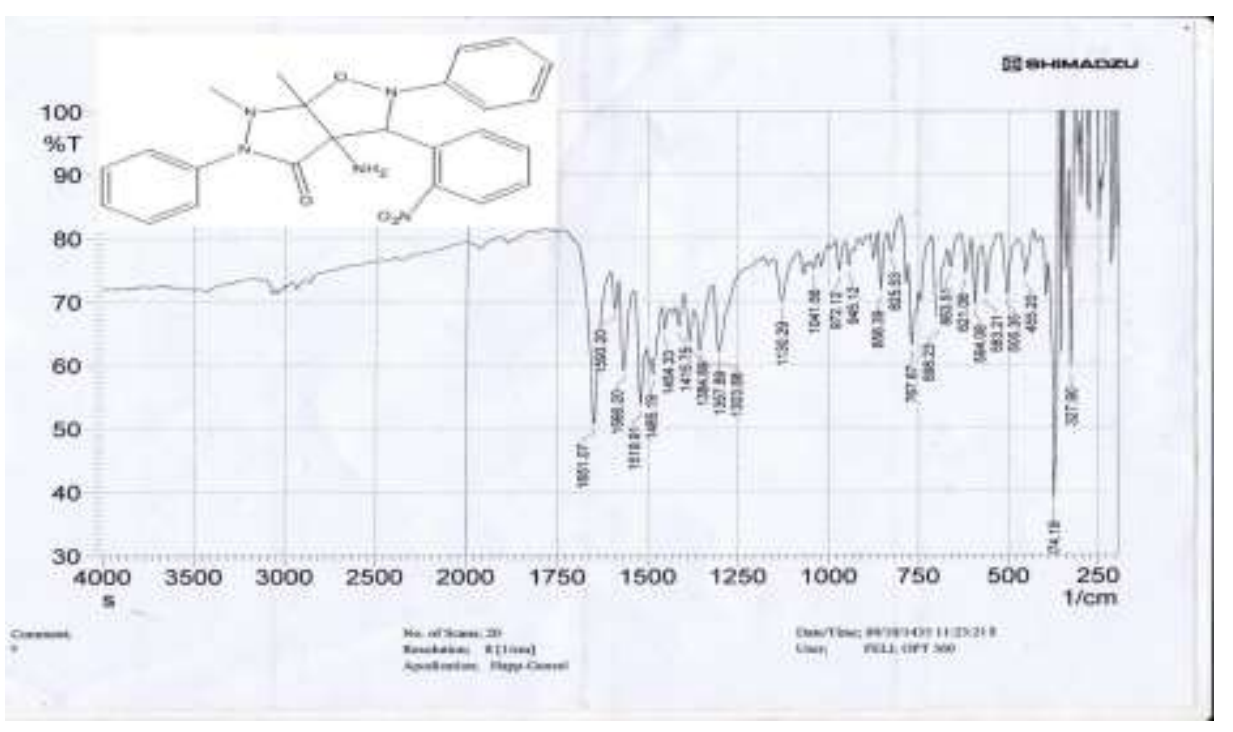

Fig. (19) IR spectrum for compound (9) 
University of Thi-Qar Journal Vol.11 No.4 DEC 2016

Web Site: https://jutq.utq.edu.iq/index.php/main Email: journal@jutq.utq.edu.iq

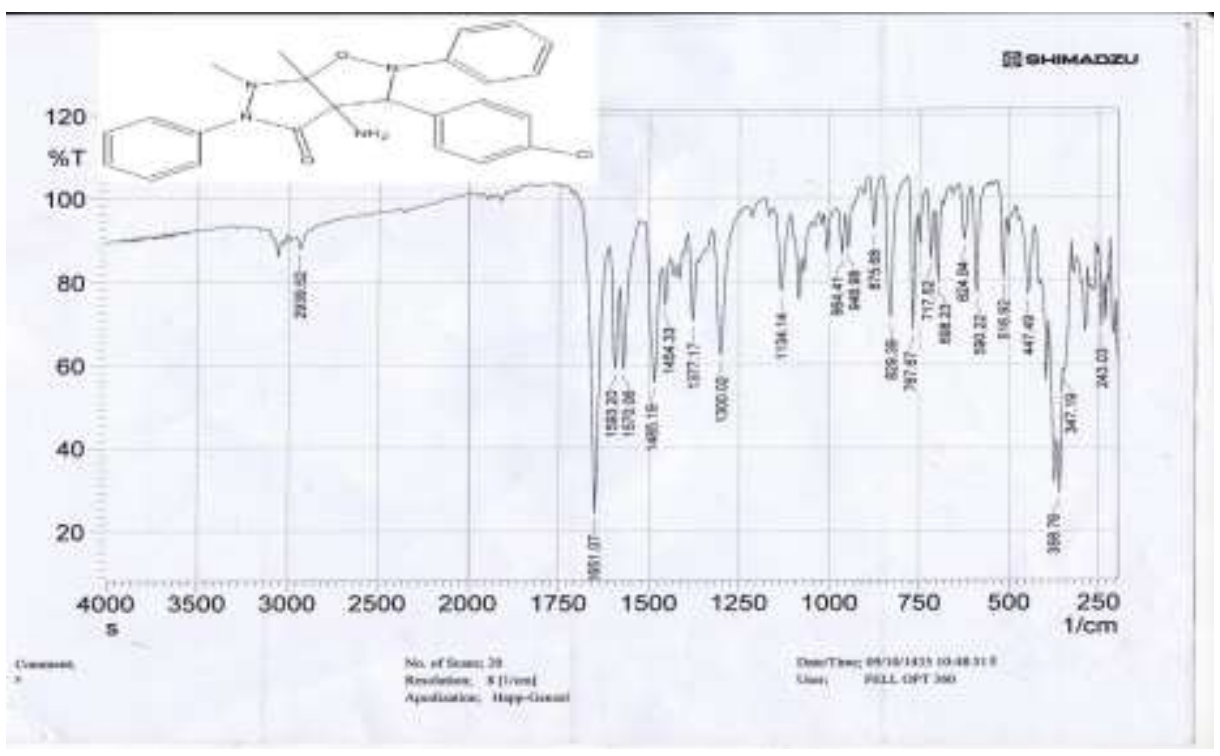

Fig. (20) IR spectrum for compound (10) 